\title{
Peptide inhibitors against influenza virus
}

\begin{abstract}
Influenza A virus is a particularly problematic virus because of its ability to cause high levels of morbidity on a global scale within a remarkably short period of time. It also has the potential to kill very large numbers of people as occurred in the Spanish influenza pandemic in 1918. Options for antiviral therapy are limited because of the paucity of available drugs and the rapid mutation rate of the virus leading to the emergence of drug-resistant strains. The current H1N1 pandemic and potential threats posed by other strains highlight the need to develop novel therapeutic and prophylactic strategies. Here, we summarize the current state and recent developments of peptide-based inhibitors of influenza A virus.
\end{abstract}

Keyword: Peptide inhibitors; Influenza virus 